\title{
Eficacia de los tratamientos ortopodológicos en pacientes con apofisitis calcánea. Revisión sistemática
}

\author{
Effectiveness of conservative treatments in patients with calcaneal apophysitis. Systematic \\ review
}

\author{
Marta Giménez Martínez ${ }^{1}$, Laura Ramos Petersen ${ }^{1,2}$, Manuel Mosqueira Ouréns ${ }^{1,2}$ y \\ Andrés López del Amo Lorente ${ }^{1,2}$ \\ 'Departamento de Podología. Universidad Católica San Antonio de Murcia. España. ${ }^{2}$ Grupo de Investigación Avances en Biomecánica Deportiva \\ y Ortopodología (ABIDOR). Universidad Católica San Antonio de Murcia. España
}

\section{Palabras clave:}

Apófisis calcánea, enfermedad de Sever, plantillas, ortesis plantares, cazoleta, dolor.
Keywords:

Calcaneal apophysitis, Sever's disease, insole, foot orthoses, heel cup, pain.

\section{Resumen}

Objetivos: La apofisitis calcánea es una lesión musculoesquelética común en niños de 8-15 años. Representa entre el 2-16 \% de las consultas en clínicas pediátricas. Se manifiesta con dolor y sensibilidad localizada en la región posterior e inferior del calcáneo en pacientes físicamente activos o con obesidad. El objetivo principal de este estudio es conocer el nivel de eficacia de los tratamientos ortopodológicos en pacientes con apofisitis calcánea.

Material y métodos: Se ha llevado a cabo una revisión sistemática siguiendo el método PRISMA, en bases de datos como Cochrane Library, CINAHL, DOAJ, IBECS, LILACS, PEDro, PUBMED, sciELO y Web of Science, utilizando los descriptores: "foot orthoses", "quality of life", "pain", "orthotic devices". La búsqueda fue realizada entre febrero y abril de 2020. Para evaluar el nivel de evidencia metodológica de los artículos incluidos se empleó la escala Risk of Bias de Cochrane. Los artículos incluían pacientes diagnosticados de apofisitis calcánea y que fueran tratados mediante tratamiento ortopodológico. Se excluyeron aquellos estudios con pacientes que tuvieran otra patología y que fueran tratados con otro tratamiento previamente.

Resultados: Se han seleccionado un total de cinco artículos publicados entre 2011 y 2016 con un nivel de evidencia alto. E tratamiento de la apofisitis calcánea con tratamientos ortopodológicos obtuvo una reducción en las escalas del dolor entre el valor inicial y el valor final.

Conclusiones: Tanto las taloneras como las ortesis plantares a medida con cazoleta pueden ser aplicadas como tratamiento conservador para el alivio de la sintomatología del paciente sin cese de la actividad física.

\section{Abstract}

Objectives: Calcaneal apophysitis is a common musculoskeletal injury in children between 8-15 years of age. It represents between $2-16 \%$ of the consultations in paediatric clinics. Its sing and symptoms are pain and localized tenderness in the posterior and lower region of the calcaneus in physically active or obese patients. The main objective of this study is to know the level of efficacy of orthopaedic treatments in patients with calcaneal apophysitis.

Material and methods: A systematic review was carried out following the PRISMA statement. The search was conducted in Cochrane Library, CINAHL, DOAJ, IBECS, LILACS, PEDro, PUBMED, sciELO and Web of Science using the Mesh: "foot orthoses", "quality of life "," pain "," orthotic devices". The search was carried out between February and April 2020. The Cochrane Risk of Bias scale was used to assess the level of methodological evidence of the included studies. The studies included patients diagnosed with calcaneal apophysitis treated by orthopaedic treatment. Those studies with patients who had another pathology and previously treated with another treatment were excluded.

Results: A total of five articles published between 2011 and 2016 with a high level of evidence were selected. The treatment of calcaneal apophysitis with conservative treatments showed a reduction in pain scales between the initial and the final score.

Conclusions: Both heel pad and foot orthoses with personalised heel cup are conservative treatments that relief patient's symptoms without stopping physical activity. 


\section{INTRODUCCIÓN}

La apofisitis calcánea, también conocida como enfermedad de Sever, es una lesión musculoesquelética común que fue descrita por el doctor J. W. Sever en 1912 como la presencia de dolor en el talón con sensibilidad localizada en la región posterior del calcáneo ${ }^{1-3}$. Se presenta entre las edades de 8 y 15 años ${ }^{4,5}$, aunque se ha observado en niños de incluso 6 años $^{6}$, y comprende entre el $16,3 \%$ y el $22,7 \%$ de las lesiones por sobrecarga en niños ${ }^{7}$. No existe una causa definida, aunque se han encontrado distintos motivos que provocan apofisitis calcánea, entre los que se encuentran: un periodo de rápida maduración durante el crecimiento ${ }^{8,9}$, aumento de la actividad física, obesidad ${ }^{10,11}$, microtraumatismos repetitivos relacionados con deportes de impacto ${ }^{12}$ o el acortamiento del tríceps sural ${ }^{8}$.

Las opciones de tratamiento recomendadas para la apofisitis calcánea son variadas. Diversos autores han recomendado las siguientes opciones de tratamiento: descanso o interrupción del deporte ${ }^{13-16}$, programas de ejercicios de estiramiento o fortalecimiento ${ }^{17-19}$, hielo $^{20}$, prescripción de calzado ${ }^{21} \mathrm{y}$ ortesis plantares ${ }^{1,21-23}$. Estas últimas basan su efectividad en la hipótesis de que la apofisitis calcánea es el resultado de un aumento de la tracción del tendón de Aquiles o del aumento de las fuerzas de impacto repetitivas en el talón traumatizando aún más la apófisis. James y cols. ${ }^{24}$ con su revisión sistemática y Uvelli y cols. ${ }^{25}$ con su revisión narrativa concluían que varios tratamientos conservadores para la apofisitis calcánea pueden producir mejoras moderadas a corto plazo en cuanto el dolor y la funcionalidad. El dolor relacionado con esta inflamación debe cesar después de la fusión del centro de osificación secundario del calcáneo.

La apofisitis calcánea es una lesión musculoesquelética que representa un alto porcentaje de las visitas pediátricas en las clínicas podológicas; a pesar de ello, existen pocos ensayos clínicos en los últimos años sobre la eficacia de los tratamientos ortopodológicos, por lo que se nos plantea la necesidad de realizar una rigurosa revisión sistemática con el objetivo de actualizar y conocer la eficacia de la disminución del dolor a través de los tratamientos ortopodológicos en pacientes con apofisitis calcánea.

\section{MATERIAL Y MÉTODOS}

Se realizó una revisión sistemática basada en los criterios expuestos según la normativa "Prefered reported ítems of systematic reviews and meta-analysis" (PRISMA) ${ }^{26}$ con el objetivo de minimizar el riesgo de cometer sesgo de publicación y de selección, y para asegurar el correcto proceso metodológico.

Se realizó una búsqueda en las bases de datos y plataformas científicas más relevantes en el ámbito de ciencias de la salud. Se consultaron: Cochrane Library, CINAHL, DOAJ, IBECS, LILACS, PEDro, PubMed, sciELO y Web of Science, y plataformas digitales como ScienceDirect o Elsevier desde la primera versión disponible hasta abril de 2020. Los estudios fueron seleccionados según la estrategia PICO (Tabla I).

Los términos de búsqueda utilizados como descriptores fueron "foot orthoses", "quality of life", "pain", "orthotic devices". Como términos en lenguaje natural, términos libres o sinónimos usados fueron "calcaneal apophysitis", "Sever's disease", "Sever's injury", "cup heel", "heel lift", "insole". Fueron combinados con los operadores lógicos o booleanos "AND" y "OR".

La estrategia de búsqueda empleada en PubMed y Cochrane Library es la siguiente: (( "calcaneal apophysitis" OR "Sever's disease" OR "Sever's injury")) AND (insole OR foot orthoses [MesH] OR Orthotic Devices [MesH] OR Cup heel OR Heel lift) AND (Quality of life [MesH] OR Pain [MesH])). Para PubMed y Cochrane Library se utilizaron tanto los descriptores (Mesh) como los términos libres. Para el resto de bases de datos y plataformas digitales se usaron solo los términos libres o sinónimos. La búsqueda fue llevada a cabo entre febrero y abril de 2020.

En esta revisión sistemática los criterios establecidos para la inclusión de los artículos fueron: ensayos clínicos controlados aleatorizados, artículos en inglés y español, los sujetos debían ser pacientes diagnosticados de apofisitis calcánea y tratados mediante tratamiento ortopodológico para la mejora de dicha patología. Y los criterios establecidos para la exclusión fueron: pacientes que tuvieran otra patología como fractura, tumor o artritis, y pacientes tratados con otro tratamiento previamente.

La selección de los estudios se llevó a cabo por un solo investigador. Para responder a los objetivos propuestos se procedió a realizar un cribado realizando la lectura del título y resumen y descartando los que no trataban sobre la patología motivo de estudio. A continuación se hizo una lectura a texto completo de los artículos elegidos hasta el momento. Para evaluar el nivel de la calidad metodológica de los artículos incluidos se empleó la escala Risk of Bias de Cochrane $(\operatorname{RoB} 2)^{27}$.

\section{RESULTADOS}

Con las estrategias de búsqueda empleadas se identificaron un total de 46 referencias, de las cuales, tras la eliminación de los duplicados, se obtuvo un total de 14 artículos como poten-

\begin{tabular}{l|l}
\multicolumn{2}{|l}{ Tabla I. Estrategia PICO. } \\
\hline Patient (Pacientes) & $\begin{array}{l}\text { Pacientes con apofisitis } \\
\text { calcánea }\end{array}$ \\
\hline Intervention (Intervención) & Tratamiento ortopodológico \\
\hline Comparison (Comparación) & $\begin{array}{l}\text { Otro tipo de intervención con } \\
\text { tratamiento conservador }\end{array}$ \\
\hline Outcome (Resultados) & Disminución del dolor \\
\hline
\end{tabular}




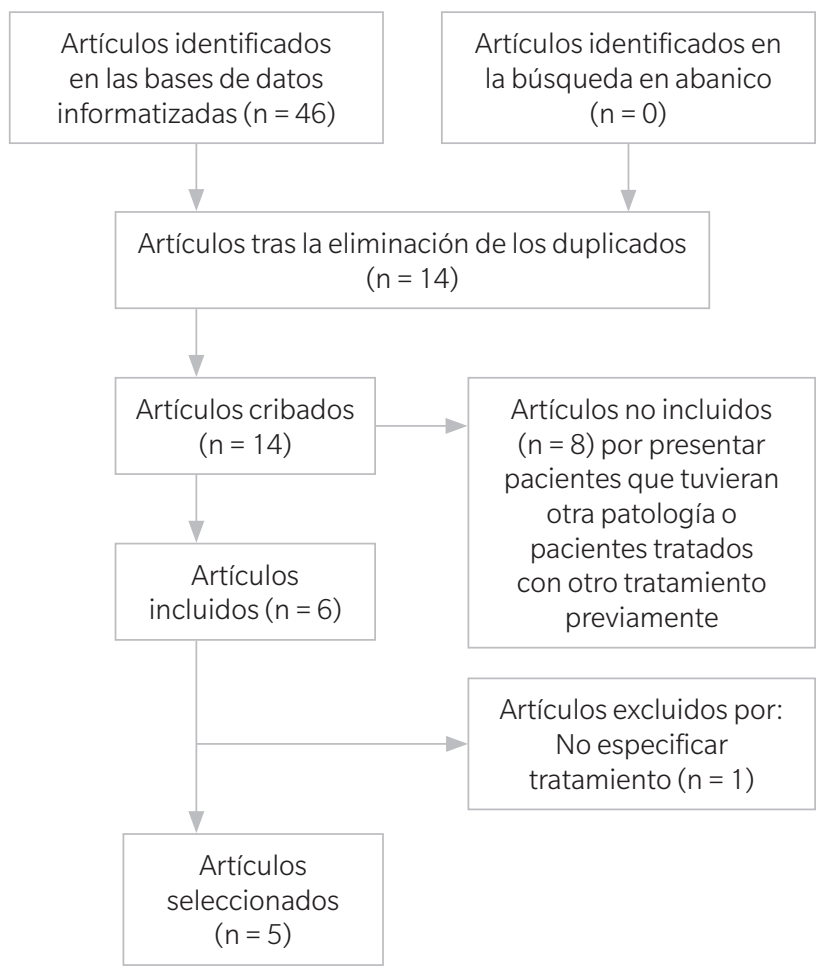

Figura 1. Flujograma.

cialmente relevantes. A continuación se excluyeron 8 artículos por no cumplir con los criterios de inclusión y exclusión, y se descartó 1 artículo por no especificar concretamente el tratamiento administrado. Finalmente, fueron 5 los artículos seleccionados para realizar esta revisión sistemática (Figura 1).

La calidad metodológica de los estudios revisados muestra que el $60 \%$ de los artículos obtuvieron la puntuación máxima de valoración (7/7) y el $40 \%$ restante obtuvieron $5 / 7$ y $6 / 7$, de manera que se puede afirmar que la calidad metodológica y su fiabilidad clínica son buenas (Figura 2).

El tamaño total de la muestra inicial es de 357 sujetos. Un total de 11 pacientes abandonaron los estudios por diversos motivos, por lo que la muestra final fue de 346 sujetos. De ellos, 284 formaron parte del grupo experimental y 62 del grupo control. Respecto al sexo, el número total de varones fue notablemente superior al de mujeres y la edad de los participantes oscila entre 8 y 15 años, siendo muy homogénea en todos los estudios. En concreto, la media de todos los ensayos es de 11.11 años (Tabla II).

El propósito del estudio de Wiegerincky cols. ${ }^{28}$ fue determinar el tratamiento conservador más eficaz para la disminución del dolor en la apofisitis calcánea. Presentó tres grupos, de los que el primero era un grupo control o grupo sin tratamiento ("esperar y ver"), el segundo recibió tratamiento mediante una talonera, en este caso con ViscoHeel de Bauerfeind, y el tercero recibió tratamiento de fisioterapia. Los resultados de este estudio demuestran claramente que cada una de las opciones de tratamiento conservador estudiadas era igualmente efec-

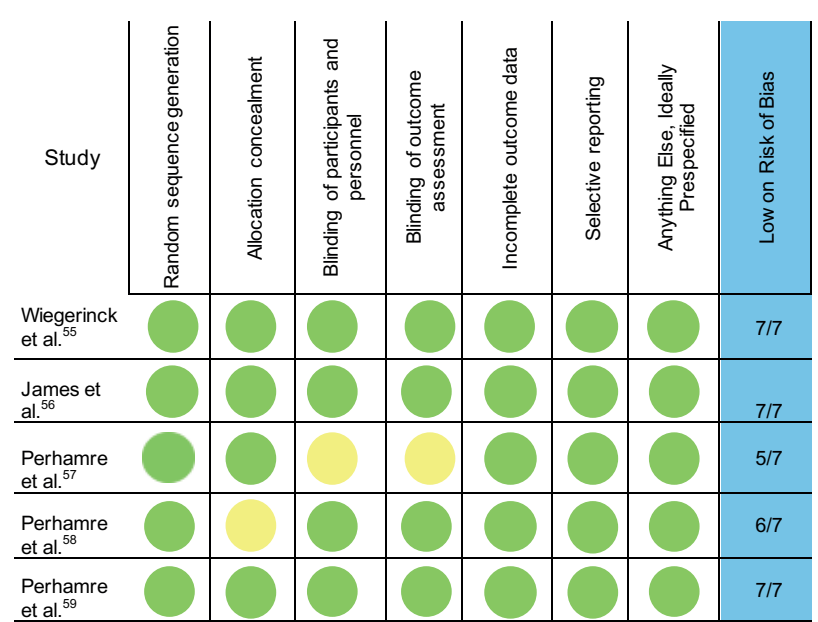

Figura 2. Calidad metodológica y evaluación del riesgo de sesgo Cochrane en los estudios incluidos.

tiva a los 3 meses de seguimiento. A las 6 semanas de seguimiento, se encontró una pequeña diferencia significativa de 4 puntos en la escala Oxford Ankle Foot Questionnaire Children (OxAFQ-C) entre el grupo de "esperar y ver" y el grupo de talonera $(p=0,009)$, y una diferencia significativa entre el grupo de "esperar y ver" y el de fisioterapia $(p=0,004)$ a favor de la fisioterapia. La única diferencia estadísticamente significativa y clínicamente relevante es la "satisfacción con el tratamiento recibido" a las 6 semanas de seguimiento. La satisfacción del paciente en el grupo de talonera fue significativamente superior en comparación con el grupo de fisioterapia y el grupo de "esperar y ver" a las 6 semanas ( $p=0,01$ y $p=0,001$, respectivamente). Se observó una preferencia por el uso de talonera debido a su falta de desventajas y a su bajo coste.

En el estudio de James y cols. ${ }^{29}$ se incluyeron cuatro grupos de tratamiento. El primer grupo experimental trataba la combinación del calzado habitual del niño añadiéndole una talonera de $6 \mathrm{~mm}$; al segundo grupo se le dio una zapatilla deportiva nueva y además se le añadió también la talonera de $6 \mathrm{~mm}$; en un tercer grupo los niños llevaban su calzado habitual y se incluyó una ortesis prefabricada de poliuretano, y un último grupo combinó una zapatilla deportiva nueva con una ortesis prefabricada de poliuretano. En los resultados obtenidos hubo un efecto principal de la talonera para los niños en el dominio físico y escolar de la escala de calidad de vida OXAFQ-C. A los 6 y 12 meses no se identificó ningún cambio relativo en ninguno de los enfoques de tratamiento, por lo que se concluye que el uso de talonera en las etapas iniciales de intervención puede ayudar a los niños a mantener la actividad física en comparación con el uso de ortesis prefabricada.

En el estudio de Perhamre y cols. (2011) ${ }^{30}$ se mostraron dos grupos de tratamiento: el primer grupo usó una ortesis con talonera de $5 \mathrm{~mm}$ de corcho y en segundo grupo una ortesis con cazoleta de termoplástico rígido con un borde de $2-3 \mathrm{~cm}$ y soporte del arco plantar moldeado sobre el pie. Concluye- 


\begin{tabular}{|c|c|c|c|c|c|c|c|c|c|}
\hline \multirow{2}{*}{ Autor/e } & \multirow{2}{*}{ Muestra } & \multicolumn{3}{|c|}{ Pie afectado } & \multicolumn{2}{|l|}{ Sexo } & \multirow{2}{*}{$\begin{array}{l}\text { Edad (SD) } \\
\text { (años) }\end{array}$} & \multirow{2}{*}{$\begin{array}{l}\text { Escala: dolor inicial } \\
\text { (SD) }\end{array}$} & \multirow{2}{*}{$\begin{array}{l}\text { Tiempo de } \\
\text { evolución } \\
\text { (meses) }\end{array}$} \\
\hline & & Izquierdo & Derecho & Bilateral & M & $\mathrm{H}$ & & & \\
\hline \multirow{3}{*}{$\begin{array}{l}\text { Wiegerinck, } \\
\text { y cols. (28) }\end{array}$} & GC: 32 & 12 & 20 & \multirow{3}{*}{ N/A } & \multirow{3}{*}{$25 \%$} & \multirow{3}{*}{$75 \%$} & $10.9(1.9)$ & FPS-R 6.1 (1.9) & \multirow{3}{*}{1} \\
\hline & GE1: 33 & 17 & 16 & & & & $10.3(1.4)$ & FPS-R 6.2 (2.0) & \\
\hline & GE2: 33 & 12 & 21 & & & & $10.6(1.4)$ & FPS-R 6.3 (1.7) & \\
\hline \multirow{4}{*}{$\begin{array}{l}\text { James, } \\
\text { y cols. (29) }\end{array}$} & GE1: 31 & 6 & 0 & 25 & \multirow{4}{*}{$42 \%$} & \multirow{4}{*}{$58 \%$} & $11.20(1.58)$ & \multirow{4}{*}{$\mathrm{N} / \mathrm{A}$} & 10.08 \\
\hline & GE2: 31 & 6 & 0 & 25 & & & $10.61(1.36)$ & & 11.52 \\
\hline & GE3: 31 & 5 & 1 & 25 & & & $10.90(1.56)$ & & 11.64 \\
\hline & GE4: 31 & 1 & 0 & 30 & & & $10.80(1.41)$ & & 9.36 \\
\hline \multirow{2}{*}{$\begin{array}{l}\text { Perhamre } 1, \\
\text { y cols. (30) }\end{array}$} & GE1: 20 & \multirow{2}{*}{9} & \multirow{2}{*}{10} & \multirow{2}{*}{25} & \multirow{2}{*}{$0 \%$} & \multirow{2}{*}{$100 \%$} & 12 & Borgs (CR-10 4 & 2 \\
\hline & GE2: 24 & & & & & & 12 & Borgs (CR-10 5 & 3 \\
\hline \multirow{3}{*}{$\begin{array}{l}\text { Perhamre, } \\
\text { y cols. (31) }\end{array}$} & GC1: 15 & \multirow{3}{*}{6} & \multirow{3}{*}{5} & \multirow{3}{*}{24} & \multirow{3}{*}{$20 \%$} & \multirow{3}{*}{$80 \%$} & \multirow{3}{*}{11} & $\mathrm{~N} / \mathrm{A}$ & \multirow{3}{*}{0.5} \\
\hline & GC2: 15 & & & & & & & Borgs (CR-10 7 & \\
\hline & GE1: 15 & & & & & & & Borgs (CR-10 7 & \\
\hline \multirow{2}{*}{$\begin{array}{l}\text { Perhamre 2, } \\
\text { y cols. (32) }\end{array}$} & GE1: 17 & \multirow{2}{*}{18} & & \multirow{2}{*}{17} & \multirow{2}{*}{$0 \%$} & \multirow{2}{*}{$100 \%$} & 17 & Borgs (CR-10 4.5 & \\
\hline & GE2: 18 & & & & & & & Borgs (CR-10 4 & \\
\hline
\end{tabular}

Borg's CR-10: escala de Borg CR-10 de medición del dolor. FPS-R: Faces Pain Scale-Revised. GC: grupo control. GE: grupo experimental. H: hombres. M: mujeres. N/A: no aparece. SD: desviación estándar.

ron que el dolor (medido con la escala CR-10 de Borg, que es una escala EVA con anclas de explicaciones verbales agregadas a los números de la escala de dolor) fue significativamente menor en el grupo de la cazoleta en comparación con el de la talonera, lo que indica que la reducción de las fuerzas de impacto repetitivas en el golpe del talón es el factor más importante para el alivio del dolor. Después de 26 semanas de intervención, cuando concluyó el estudio, los niños pudieron elegir en una segunda fase el tratamiento. Para un $77 \%$ de los niños la elección fue la cazoleta, frente a un $23 \%$ que eligieron la talonera, aunque parece obvio que ambas ortesis ofrecieron alivio del dolor.

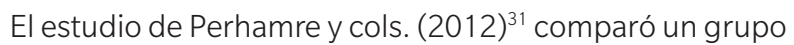
control en el que se estudiaba a los sujetos sin tratamiento (descalzos), frente a un grupo con zapatilla deportiva y otro con zapatilla deportiva más cazoleta. Obtuvieron que el grosor de la grasa plantar del talón aumentó significativamente en 2,19 mm cuando se usó calzado deportivo solo en comparación con el pie descalzo $(p<0,001)$. Añadir la cazoleta al zapato deportivo incrementó significativamente el grosor de la grasa plantar del talón en $1,43 \mathrm{~mm}$ más $(p=0,002)$. Por lo tanto, el grosor de la grasa plantar del talón se incrementó en $3,57 \mathrm{~mm}$ cuando se usaba un zapato deportivo con una cazoleta en comparación con estar descalzo $(p<0,001)$. Este estudio mostró una reducción significativa del dolor durante las actividades deportivas en el grupo de tratamiento con cazoleta durante el periodo de estudio. El grupo sin tratamiento reflejó una reducción del dolor según el curso natural (de 7,0 al inicio del estudio a 5,0 después de las 4 semanas).

Por último, Perhamre y cols. $(2011)^{32}$, en su investigación, incluyeron un grupo con ortesis con cazoleta de plástico rígido moldeado sobre el pie con 2-3 cm de altura y que incluyó soporte del arco plantar, y un segundo grupo con ortesis con talonera de $5 \mathrm{~mm}$ de corcho. Este estudio mostró que el tratamiento con dos tipos diferentes de ortesis, una talonera y una cazoleta, proporcionaba un alivio efectivo del dolor de talón en un grupo de niños físicamente activos, sin una reducción en su actividad física. Hubo una reducción significativa en el nivel de dolor durante la actividad con la ortesis con cazoleta y la ortesis con talonera, al comparar los niveles de dolor en la fase de pretratamiento y tratamiento, para las actividades $A$ (fútbol) y $\mathrm{B}$ (carrera) $(p<0,001)$. Después de quitar la ortesis, el nivel de dolor aumentó en la actividad física. Esto demuestra que la reducción del dolor es un efecto del tratamiento y no se debe a la curación espontánea como parte del curso natural (Tabla III).

Para completar las intervenciones explicadas anteriormente, los autores añadieron en algunos casos un tratamiento complementario a sus grupos. En el caso de Wiegerinck y cols. ${ }^{28}$, en el grupo de tratamiento de control se explicó a los 
Tabla III. Características de la intervención.

\begin{tabular}{|c|c|c|c|c|}
\hline Autor/es & Tratamiento & TTO. Complementario & Duración & Revisiones \\
\hline \multirow{3}{*}{$\begin{array}{l}\text { Wiegerinck, } \\
\text { y cols. (28) }\end{array}$} & GC: $\sin$ TTO & $\begin{array}{l}\text { Cese de actividad } \\
\text { deportiva }\end{array}$ & 10 & 1.5 y 3 \\
\hline & GE1: talonera (viscoheel Bauerfeind) & $N / A$ & & \\
\hline & GE2: fisioterapia & Ejercicios diarios en casa & & \\
\hline \multirow{4}{*}{$\begin{array}{l}\text { Wiegerinck, } \\
\text { y cols. ( } 29)\end{array}$} & GE1: calzado usual y talonera de $6 \mathrm{~mm}$ & \multirow{4}{*}{$\begin{array}{l}\text { Estiramientos + Terapia } \\
\text { de hielo }\end{array}$} & \multirow{4}{*}{52} & \multirow{4}{*}{$0,1,2,6$ y 12} \\
\hline & GE2: calzado deportivo nuevo y talonera de $6 \mathrm{~mm}$ & & & \\
\hline & GE3: calzado usual y ortesis prefabricada de poliuretano & & & \\
\hline & GE4: calzado deportivo nuevo y ortesis prefabricada de poliuretano & & & \\
\hline \multirow{2}{*}{$\begin{array}{l}\text { Perhamre } 1, \\
\text { y cols. }(30)\end{array}$} & GE1: ortesis con talonera de $5 \mathrm{~mm}$ & \multirow[b]{2}{*}{$\mathrm{N} / \mathrm{A}$} & \multirow[b]{2}{*}{26} & \multirow{2}{*}{$\begin{array}{l}0,0.5,1.5,2 \\
3,3.5 \text { y } 6\end{array}$} \\
\hline & $\begin{array}{l}\text { GE2: ortesis con cazoleta de termoplástico rígido con borde de } \\
2-3 \mathrm{~cm} \text {, moldeada sobre el talón con soporte del arco plantar }\end{array}$ & & & \\
\hline \multirow{3}{*}{$\begin{array}{l}\text { Perhamre 1, } \\
\text { y cols. (31) }\end{array}$} & GC1: descalzo & \multirow{3}{*}{$\mathrm{N} / \mathrm{A}$} & \multirow{3}{*}{4} & \multirow{3}{*}{0.1} \\
\hline & GC2: zapatilla deportiva & & & \\
\hline & GE1: zapatilla deportiva con ortesis con cazoleta & & & \\
\hline $\begin{array}{l}\text { Perhamre 2, } \\
\text { y cols. (32) }\end{array}$ & $\begin{array}{l}\text { GE1: ortesis con cazoleta de plástico rígido moldeado de } 2-3 \mathrm{~cm} \\
\text { con soporte del arco }\end{array}$ & $\mathrm{N} / \mathrm{A}$ & 4 & $\mathrm{~N} / \mathrm{A}$ \\
\hline
\end{tabular}

GC: grupo control. GE: grupo experimental. N/A: no aparece. TTO: tratamiento.

sujetos que debían cesar la actividad deportiva que realizaban en el momento en que esta produjese dolor; en el grupo experimental con talonera se explicó que podían continuar con su actividad física, y un segundo grupo experimental de fisioterapia añadió ejercicios diarios en casa. James y cols. ${ }^{29}$, en todos sus grupos de intervención, añadió una terapia de hielo de 10 minutos en la zona de dolor una vez al día en la etapa inicial (1 mes), y hielo tras actividad deportiva hasta que no se sintiera dolor y estiramientos durante 1 mes después de que la fase aguda del dolor disminuyera, siendo del tipo de estiramientos estáticos de gastrocnemios y sóleo. En los tres ensayos restantes se expresó que debían continuar con sus altos niveles de actividad, sin añadir ningún tratamiento complementario.

\section{DISCUSIÓN}

El tratamiento conservador está dirigido a disminuir el proceso inflamatorio y la tensión musculoesquelética y a eliminar los microtraumatismos de repetición. Para ello se usan ortesis plantares con cazoleta alta para concentrar la grasa plantar del calcáneo consiguiendo una amortiguación de talón o con elevación de talón a fin de disminuir las fuerzas de reacción del suelo.
Los hallazgos encontrados en esta revisión sobre la eficacia de los tratamientos ortopodológicos para la apofisitis calcánea coinciden con la evidencia disponible ${ }^{24,33}$ de que los tratamientos conservadores con ortesis plantares usados por los autores (talonera, cazoleta, ortesis prefabricada) mejoran la sintomatología dolorosa. James y cols. ${ }^{24}$, en su revisión sistemática, indicaron que las ortesis con cazoleta y el soporte del arco medial son más efectivos para reducir el dolor en las actividades deportivas al compararlos con la talonera o con la falta de tratamiento. Pero se demuestra que la talonera también reduce el dolor en las actividades deportivas al compararla con ningún tratamiento.

De los cinco ensayos clínicos aleatorizados revisados en este estudio, solo el de Perhamre y cols. $(2011)^{30}$ coincide con las afirmaciones aportadas por James y cols. ${ }^{24}$ acerca de que las ortesis con talonera y el soporte del arco medial son más efectivos para disminuir el dolor en las actividades deportivas frente al tratamiento de talonera o la falta de tratamiento. Sin embargo, esto no se confirma en el resto de estudios dado que el resto de autores o no comparan entre sí los dos grupos experimentales de tratamiento con talonera y tratamiento con cazoleta, o no incluyen ambos tratamientos dentro del ensayo.

Se observa que las ortesis producen un alivio del dolor a corto plazo, concretamente entre los meses 1 y 2 de trata- 
miento. Retirar al paciente el tratamiento una vez eliminada la sintomatología dolorosa puede provocar recidivas, tal y como indica Perhamre y cols. $(2011)^{32}$. Este autor observó un aumento del dolor 2 semanas después de retirar las ortesis plantares. Estos resultados coinciden con los obtenidos por Medina y cols. ${ }^{9}$, quienes reflejan que esta patología puede producir recidivas que se resolverán por completo cuando finalice el crecimiento y el desarrollo del centro de osificación secundario. Destacamos, al igual que el resto de los autores, la importancia de mantener el tratamiento ortopodológico hasta 12 meses después de desaparecer la sintomatología dolorosa, para evitar recidivas.

Los cinco autores revisados no explican o aclaran la toma de molde ni el proceso de fabricación de una ortesis plantar a medida. Autores como Gijón y cols. ${ }^{34}$ concluían que las ortesis plantares hechas a medida (cazoleta y un soporte del arco plantar) y fabricadas con resinas de PVC y poliéster combinadas con una cazoleta y amortiguación de talón de 22 Shore A pueden reducir el dolor de la enfermedad de Sever. El conocimiento de dicha información es necesario para valorar con mayor criterio la eficacia de los distintos tratamientos ortopodológicos.

Ningún artículo tuvo en cuenta alteraciones biomecánicas asociadas a la apofisitis calcánea y solo se tuvo en cuenta como causa principal para el tratamiento conservador con ortesis plantares la sintomatología. Una postura normal del pie y una biomecánica normal del pie pueden requerir únicamente una elevación del talón para aliviar el dolor. Sin embargo, el niño que tiene cambios adicionales en la postura del pie puede necesitar algún tipo de ortesis para mejorar la función del pie. Solo James y cols. ${ }^{28}$, en sus criterios de exclusión, tienen en cuenta la morfología del pie, descartando aquellos pies con un FPI (Foot Posture Index) menor o igual a -1. Es necesario tener en consideración las posibles alteraciones biomecánicas del pie para darle tratamiento ortopodológico realizando una ortesis plantar a medida, donde se trate a la vez la apofisitis calcánea y las posibles alteraciones biomecánicas.

Dentro de las limitaciones, debemos tener en cuenta que, de los cinco ensayos clínicos incluidos para esta revisión sistemática, el 60 \% corresponden a un mismo autor (Perhamre), dos de 2011 y otro trabajo de 2012.

Debido a la baja evidencia científica sobre la eficacia de los tratamientos ortopodológicos para la apofisitis calcánea en niños, consideramos que los resultados obtenidos en esta revisión deben ser tomados con cautela.

Desde un punto de vista ético, entendemos cuestionable no abandonar un grupo sin ningún tratamiento y hacer que continúe en el mismo alto nivel de actividad, con un dolor considerable. Se precisan futuras líneas de investigación en las que exista un grupo control sin tratamiento y uno o varios grupos experimentales que sea o sean tratados solo con ortesis plantares, que comparen distintos tratamientos ortopodológicos entre sí y en las que se detalle tanto el proceso de fabricación de la ortesis plantar como los materiales que la conforman, para así poder tener un mayor conocimiento de la eficacia de los tratamientos ortopodológicos en pacientes con apofisitis calcánea.

Dado que no hemos obtenido diferencias claras entre los distintos tratamientos ortopodológicos, y debido a la diferencia de coste entre ellos (talonera estándar, plantilla a medida, plantilla prefabricada), es importante que en aquellas poblaciones con un nivel socioeconómico bajo se opte por el uso de taloneras en relación con el coste-beneficio, ya que el coste de las cazoletas de ortesis a medida puede ser muy superior.

Los resultados obtenidos sobre la eficacia de talonera o cazoleta en ortesis plantares a medida para el tratamiento conservador de pacientes con apofisitis calcánea mejoran la sintomatología del dolor. Cualquiera de los dos tratamientos, cazoleta o talonera, debe ser aplicado para el alivio de la sintomatología del paciente sin que por ello cese su actividad física.

\section{CONFLICTO DE INTERESES}

Los autores declaran no tener ningún conflicto de intereses.

\section{FINANCIACIÓN}

Ninguna.

\section{BIBLIOGRAFÍA}

1. Sever JW. Apophysitis of the os calcis. NY Med J. 1912;95:1025.

2. Micheli LJ, Fehlandt AF. Overuse injuries to tendons and apophyses in children and adolescents. Clin Sport Med. 1992,11:713-26. DOI: 10.1016/S0278-5919(20)30480-4.

3. Lesiones deportivas frecuentes. Acta Pediátr Costarric. 2003;17(2):65-80.

4. Ogden JA, Ganey TM, Hill JD, Jaakkola JI. Sever's Injury: a stress fracture of the immature calcaneal metaphysis. J Pediatr Orthop. 2004;24:48892. DOI: 10.1097/01241398-200409000-00007.

5. Kvist MH, Heinonen OJ. Calcaneal apophysitis (Sever's disease): a common cause of heel pain in young athletes. Scand J Med Sci Sports. 1991;1(4):235-8.

6. Volpon JB, de Carvalho Filho G. Calcaneal apophysitis: a quantitative radiographic evaluation of the secondary ossification center. Arch Orthop Trauma Surg. 2002;122(6):338-41. DOI: 10.1007/s00402-002-0410-y.

7. Becerro-de-Bengoa-Vallejo R, Losa-Iglesias ME, Rodríguez-Sanz D. Static and dynamic plantar pressures in children with and without Sever disease: A case-control study. Physical Therapy. 2014;94 (6):818-26. DOI: 10.2522/ptj.20120164.

8. Alfaro Santafé J, Gómez Bernal A, Alfaro Santafé JV, Lanuza Cerzócimo C, Escamilla Galindo VL, Almenar Arasanz AJ. Coalición calcáneo-navicular asociada a pie plano rígido infantil. Relación de Lunge y Jack Test en la apófisis calcánea (talalgia de Sever) en futbolistas jóvenes. Rev Int Cienc Podol. 2017;11(2):117-23. DOI: 10.5209/RICP.56028.

9. Micheli LJ, Ireland ML. Prevention and management of calcaneal apophysitis in children: an overuse syndrome. J Pediatr Orthop. 1987;7:34-8. DOI: 10.1097/01241398-198701000-00007.

10. Espinosa Leal EA, Espinosa Hernández EA. Síndrome de talón doloroso, enfermedad de Sever: presentación clínica, hallazgos de imágenes y manejo del dolor en niños y jóvenes atletas. Rev Méd Costa Rica Centroam. 2016;73(619):383-7.

11. Hernández García S, Martínez Couce I, Menéndez Fadraga L, Sosa Fernández A, Rodríguez Arencibia MÁ. Resultados del tratamiento homeopático de la enfermedad de Sever. Rev Cienc Méd Pinar Río. $2016 ; 20(2): 44-53$ 
12. James AM, Williams CM, Luscombe M, Hunter R, Haines TP. Factors associated with pain severity in children with calcaneal apophysitis (Sever disease). J Pediatr. 2015;167(2):455-9. DOI: 10.1016/j. jpeds.2015.04.053.

13. Pose LG. Lesiones deportivas osteocartilaginosas en el niño y adolescente. Rev Chil Radiol. 2005;11(2):91-100. DOI: 10.4067/S071793082005000200008.

14. Ebri JR. El pie infantil: crecimiento y desarrollo. Deformidades más frecuentes: pie doloroso. Pediatr Integral. 2002;6(5):431-52.

15. Dautt Medina PM, Vidal Ruiz CA, Íñiguez R, Martínez A, Del Carmen A. Sever disease or calcaneal apophysitis. A pathology not well identified. Rev Mex Ortop Ped. 2019;21(1-3):18-21.

16. Rodríguez-Sanz D, Becerro-de-Bengoa-Vallejo R, López-López D, et al. Slow velocity of the center of pressure and high heel pressures may increase the risk of Sever's disease: a case-control study. BMC Pediatr. 2018;18(1):357. DOI: 10.1186/s12887-018-1318-1.

17. Lee KT, Young KW, Park YU, Park SY, Kim KC. Neglected Sever's disease as a cause of calcaneal apophyseal avulsion fracture: case report. Foot Ankle Int. 2010,31(8):725-8. DOI: 10.3113/FAl.2010.0725.

18. Clemow C, Pope B, Woodall HE. Tools to speed your heel pain diagnosis: Quickly zero in on a diagnosis by using our handy "photo guide" and reference table. J Fam Pract. 2008;57(11):714-21.

19. Toomey EP. Plantar heel pain. Foot Ankle Clin. 2009;14(2):229-45. DOI: 10.1016/j.fcl.2009.02.001.

20. Rehbock DS. Some of the more common biomechanical and orthopaedic foot conditions that are treated by the podiatrist. S Afr Pharm J. 2009;76(3):35-42.

21. Micheli LJ, Ireland ML. Prevention and management of calcaneal apophysitis in children: an overuse syndrome. J Pediatr Orthop. 1987;7(1):348. DOI: 10.1097/01241398-198701000-00007.

22. Dalgleish M. Calcaneal apophysitis [Sever's disease] clinically based treatment. Sports Med News. 1990. June 15.

23. Kuyucu E, Gülenç B, Biçer H, Erdil M. Assessment of the kinesiotherapy's efficacy in male athletes with calcaneal apophysitis. J Orthop Surg Res. 2017;12(1):146. DOI: 10.1186/s13018-017-0637-5.

24. James AM, Williams CM, Haines TP. Effectiveness of interventions in reducing pain and maintaining physical activity in children and adoles- cents with calcaneal apophysitis (Sever's disease): a systematic review. J Foot Ankle Res. 2013;6(1):16. DOI: 10.1186/1757-1146-6-16.

25. Uvelli K, Neher JO, Safranek S. Treatment for calcaneal apophysitis. Am Fam Physician. 2017;96(2):126-7.

26. Urrutia G, Bonfill X. Declaración PRISMA: una propuesta para mejorar la publicación de revisiones sistemáticas y metaanálisis. Med Clin. 2010;135(11):507-11. DOI: 10.1016/j.medcli.2010.01.015.

27. Higgins JP, Altman DG, Gøtzsche PC, Jüni P, Moher D, Oxman AD, et al. The Cochrane Collaboration's tool for assessing risk of bias in randomised trials. BMJ. 2011,343. DOI: 10.1136/bmj.d5928.

28. Wiegerinck JI, Zwiers R, Sierevelt IN, van Weert HC, van Dijk CN, Struijs PA. Treatment of calcaneal apophysitis: wait and see versus orthotic device versus physical therapy: A pragmatic therapeutic randomized clinical trial. J Pediatr Orthop. 2016;36(2):152-7. DOI: 10.1097/ BPO.0000000000000417

29. James AM, Williams CM, Haines TP. Effectiveness of footwear and foot orthoses for calcaneal apophysitis: a 12-month factorial randomised trial. Br J Sports Med. 2016;50(20):1268-75. DOI: 10.1136/ bjsports-2015-094986.

30. Perhamre S, Lundin F, Norlin R, Klässbo M. Sever's injury, treat it with a heel cup: a randomized, crossover study with two insole alternatives. Scand J Med Sci Sports. 2011;21(6):42-7. DOI: 10.1111/j.16000838.2010.01140.x

31. Perhamre S, Lundin F, Klässbo M, Norlin R. A heel cup improves the function of the heel pad in Sever's injury: effects on heel pad thickness, peak pressure and pain. Scand J Med Sci Sports. 2012;22(4):516-22. DOI: 10.1111/j.1600-0838.2010.01266.x.

32. Perhamre S, Janson S, Norlin R, Klässbo M. Sever's injury: treatment with insoles provides effective pain relief. Scand J Med Sci Sports. 2011;21(6):819-23. DOI: 10.1111/j.1600-0838.2010.01051.x.

33. Launay F. Sports-related overuse injuries in children. Orthop Traumatol Surg Res. 2015;101(1):139-47. DOI: 10.1016/j.otsr.2014.06.030.

34. Gijón-Nogueron G, Cortes-Jerónimo E, Cervera-Marín JA, García-dela-Peña R, Benhamu-Benhamu S, Luque-Suarez A. Foot orthoses custom-made by vacuum forming on the non-load-bearing foot: preliminary results in male children with calcaneal apophysitis (Sever's disease). Prosthet Orthot Int. 2013;37(6):495-8. DOI: 10.1177/0309364613482844. 